\title{
Sensitivity of medium-baseline reactor neutrino mass-hierarchy experiments to nonstandard interactions
}

\author{
Amir N. Khar* \\ Department of Physics, COMSATS IIT, Park Road, Islamabad, 44000, Pakistan \\ Douglas W. McKay \\ Department of Physics and Astronomy, University of Kansas, Lawrence, KS 66045 \\ F. Tahir \\ Department of Physics, COMSATS IIT, Park Road, Islamabad 44000, Pakistan
}

(Dated: July 31, 2018)

\begin{abstract}
We explore the impact of nonstandard interactions at source and detector on the interpretation of reactor electron neutrino disappearance experiments with short and medium baseline designs. We use the constraints from the recent results from short baseline experiments and generalize current estimates of medium baseline event rates to include charged current interactions at source and detector with standard Lorentz structure but with nonstandard flavor structure. We find that the average spectrum of observed events at a baseline of $50 \mathrm{~km}$, in the middle of the currently favored region, provides a probe of new interactions. We show that an improvement in sensitivity to nonstandard interactions is possible if combined with improved precision of input mixing parameters in independent experiments, despite ambiguity in interpretation of medium baseline data. We show that nonstandard interactions can enhance or suppress the sensitivity of experiments to the mass hierarchy, depending on the phases of the parameters and the CP-violating phase in the standard three-neutrino mixing picture.
\end{abstract}

PACS numbers:

\section{INTRODUCTION}

In the past year the Double Chooz [1], Daya Bay [2] and RENO [3] electron neutrino disappearance experiments have announced measurements of the elusive parameter $\sin ^{2}\left(2 \theta_{13}\right)$ in the vicinity of 0.1 . The significance of the measurements of Daya Bay and RENO is many standard deviations from zero, making their results a milestone in the understanding of neutrino physics. Daya Bay's result is especially interesting in that its systematic error is a factor 3 smaller than its statistical error in the first announced result and a factor 2 smaller in the second announcement with greatly increased statistics [4]. The total uncertainty is about $12 \%$ and the ultimate uncertainty estimate is $1 / 3$ of that. The plans for substantial increase in precision of these measurement and ongoing plans for increased precision from accelerator experiments beyond MINOS [5] and T2K [6] make this a good time to assess the impact on searches for new physics, such as those surveyed in Refs. 7], [8] and [9]. Short and medium baseline reactor neutrino experiments involve only vacuum oscillation and thus are ideal for the purpose of revealing effects of flavor changing nonstandard interactions (NSI) at source and detector [8], since flavor conserving NSI and their contribution to matter effects only play a significant part in analysis of higher energy, longer baseline experiments.

Though charged lepton decays put stringent bounds on many lepton flavor violating parameters in the "charged current" modes, the direct evidence against lepton flavor violation in neutrino experiments, referred to as "model independent limits", is much weaker [10]. There is now a strong effort to use the large value of $\sin ^{2}\left(2 \theta_{13}\right)$ to determine the mass hierarchy $(\mathrm{MH})$ [1] 22] in medium baseline experiments, such as the JUNO [23 25] and RENO-50 26] projects. This goal was inaccessible in the KamLand experiment [27], both because the value of $\sin ^{2}\left(2 \theta_{13}\right)$ was unknown and the statistics, though sufficient for measuring $\tan ^{2} \theta_{12}$ and $\Delta m_{21}^{2}$, were far short of measuring $\sin ^{2}\left(2 \theta_{13}\right)$ and determining the MH. It is an ideal time to combine the short baseline measurements of the value of $\sin ^{2}\left(2 \theta_{13}\right)$ and the planned reactor medium baseline, precision $\mathrm{MH}$ measurements to assess the prospects of seeing new physics. The current status of NSI, including recent work on reactor neutrinos, is reviewed in [28].

\footnotetext{
*Electronic address: amir*nawaz@comsats.edu.pk

†Electronic address: dmckay@ku.edu

‡Electronic address: farida tahir@comsats.edu.pk
} 
We implement the short baseline constraint between $\sin ^{2}\left(2 \theta_{13}\right)$ and NSI parameters inherent in "same physics at source and detector", left handed NSI to determine the conditions required in the medium baseline experiments to probe deeper into the NSI parameter space to look for new physics effects. Allowing the input value of $\sin ^{2}\left(2 \theta_{12}\right)$ to vary, we find a degeneracy between the choice of this mixing angle and the choice of NSI parameter. Given the current precision of its measurement, we find this degeneracy limits the reach of the reactor data for probing NSI to values comparable to those already achieved [10]. Higher sensitivity can be achieved as solar neutrino experiments with different physics in the source, propagation and detection chain improve precision in step with that in reactor neutrino measurements 29].

Turning to the $\mathrm{MH}$ question, we find that the discrimination between the normal hierarchy (NH) and inverted hierarchy (IH) of neutrino mass splittings is complicated in the presence of NSI, showing that the sensitivity to the $\mathrm{MH}$ can be significantly affected depending on the magnitude and sign of an NSI parameter, enhanced if positive and suppressed if negative.

In the next section we briefly review our formalism and notation, then turn to a section on short baseline and medium baseline applications and energy spectra. We follow with a survey of our results on statistical sensitivity of features of the spectra to NSI and then summarize and conclude.

\section{FORMALISM OF SOURCE AND DETECTOR NSI CONSEQUENCES FOR NEUTRINO OSCILLATIONS}

As pointed out [30] and developed [31 35] a number of years ago, the presence of lepton flavor violation at the source and detector of a neutrino beam can skew the interpretation of neutrino oscillation experiments. For example, the wrong flavor neutrino provided at the source oscillates and can provide a right flavor lepton signal at the detector, confusing a wrong signal "appearance" search, or a wrong signal at the detector, confusing a right signal "disappearance search". To begin, let us establish our notation by defining the effective four-fermion, charged current semileptonic Lagrangian appropriate for the reactor neutrino application. We restrict ourselves to the case of left-handed neutrino helicity currents and vector and axial vector quark currents and write 33 ]

$$
\mathcal{L}^{s}=2 \sqrt{2} G_{F} K_{i j}\left(\bar{l}_{i} \gamma_{\lambda} P_{L} U_{j a} \nu_{a}\right)\left[\bar{d} \gamma^{\lambda}\left(P_{L}+\beta P_{R}\right) u\right]^{\dagger}+H . c .,
$$

where indices $i, j, k$ run over flavor basis labels and $a, b, c$ over mass basis labels, and repeated indices are summed over [36. The flavor label correspondences are $e=1, \mu=2$ and $\tau=3$ and the $\mathrm{d}$ and $\mathrm{u}$ spinor fields designate down and up quarks. The coefficients $K_{i j}$ represent the relative coupling strengths for the various lepton flavor combinations. For reactor disappearance search applications, a nuclear decay provides the source of neutrinos and the inverse beta decay reaction provides the electron signal for the detector. From the form of Eq. (1D) it is apparent that the effect of the NSI, represented by the elements of the dimensionless matrix $K_{i j}$, are captured by the replacement $U_{i a} \rightarrow K_{i l} U_{l a}$ in the weak Lagrangian. In the expression for the oscillation propagation amplitude for antineutrinos of flavor $i$ at the source to produce leptons of flavor $j$ at the detector, this amounts to the replacement

$$
\bar{A}_{i j}=U_{i a} e^{-i m_{a}^{2} \frac{L}{2 E}} U_{j a}^{*} \rightarrow K_{i k} U_{k a} e^{-i m_{a}^{2} \frac{L}{2 E}} K_{j l}^{*} U_{l a}^{*} .
$$

Here the neutrino mass eigenvalues are $m_{a}$, the baseline is $L$, the propagating neutrino energy is $E$ and, in the present study, we simplify to the case $\beta=0$. The repeated indices are always taken to be summed. The corresponding expression for the neutrino beam case reads

$$
A_{i j}=U_{i a}^{*} e^{-i m_{a}^{2} \frac{L}{2 E}} U_{j a} \rightarrow K_{i k}^{*} U_{k a}^{*} e^{-i m_{a}^{2} \frac{L}{2 E}} K_{j l} U_{l a} .
$$

In matrix form, the expressions to the right of the arrows in Eq. (21) and Eq. (3) can be written compactly as

$$
\bar{A}=(K U) X(K U)^{\dagger}=K\left(U X U^{\dagger}\right) K^{\dagger}, A=\bar{A}^{T}
$$

the flavor violating interactions act as a " $K$ transformation" on the standard oscillation probability. In Eq. 4 the diagonal matrix $X$ is defined as $X \equiv \operatorname{diag}\left(\exp \left(-2 i x_{1}\right), \exp \left(-2 i x_{2}\right), \exp \left(-2 i x_{3}\right)\right)$, and $2 x_{a} \equiv m_{a}^{2} L / 2 E$. When $X=1$, the unit matrix, then $\bar{A}=K K^{\dagger} \neq 1$ in general. As we describe later, in the case of $\overline{\nu_{e}} \rightarrow \overline{\nu_{e}}$ in the approximations we adopt here, $\left(K K^{\dagger}\right)_{e e}=1$.

\section{A. Effect of source and detector lepton flavor violation on reactor $\bar{\nu}_{e}$ disappearance probability formulas}

To focus on flavor changing NSI, we develop our case with the flavor changing coefficients $K_{e \mu}$ and $K_{e \tau}$. Because the freedom to redefine the phases of coefficients is already exhausted by redefining fermion fields in the standard 
mixing matrix definition, the elements of the matrix $K$ are complex in general, and we write $K_{e \mu}=\left|K_{e \mu}\right| \exp \left(i \phi_{e \mu}\right)$ and $K_{e \tau}=\left|K_{e \tau}\right| \exp \left(i \phi_{e \tau}\right)$. Our electron antineutrino propagation amplitude now reads, taking $K_{e e}=1$,

$$
\bar{A}_{e e}=\left(U_{e a}+\left|K_{e \mu}\right| e^{i \phi_{e \mu}} U_{\mu a}+\left|K_{e \tau}\right| e^{i \phi_{e \tau}} U_{\tau a}\right) e^{-2 i x_{a}}\left(U_{e a}^{*}+\left|K_{e \mu}\right| e^{-i \phi_{e \mu}} U_{\mu a}^{*}+\left|K_{e \tau}\right| e^{-i \phi_{e \tau}} U_{\tau a}^{*}\right) .
$$

The oscillation probability factor, $\bar{P}=\bar{A}^{*} \bar{A}$ can now be computed straightforwardly. We will display only the leading order terms in $K \mathrm{~s}$ in our explicit formulas below, since the flavor violating coefficients are constrained by experimental searches to be of order 0.05 or less [10]. The results we quote are valid to accuracies better than experimental uncertainties so long as no special choices between the standard mixing, CP-violating phase $\delta$ and the NSI phases reduce the linear terms to values much less than the absolute magnitudes of the NSI parameters. Since only the real part of the parameter $K_{e e}$ contributes to the disappearance probability, and its value is bounded to be more than an order of magnitude smaller than the flavor violating parameters $K_{e \mu}$ and $K_{e \tau}$ [10], we do not include it in the first order formulas. Strictly speaking, the terms in $P_{\bar{\nu}_{e} \rightarrow \bar{\nu}_{e}}$ should be normalized, but the normalization affects only higher order terms in the $K_{i j}$ parameters [37].

Sketching the organization of the $\bar{\nu}_{e}$ disappearance propagation probability with NSI at source and detector, we write the generic form of the modulus of the propagation amplitude as

$$
\left|\bar{A}_{e e}\right|=\left|A_{11}+A_{21} e^{-2 i x_{21}}+A_{31} e^{-2 i x_{31}}\right|,
$$

where $x_{i j}=x_{i}-x_{j}=\Delta m_{i j}^{2} L / 4 E$, with $\Delta m_{i j}^{2}=m_{i}^{2}-m_{j}^{2}$. In Eq. (6), the quantities $A_{i j}$ are all real. Judiciously using double angle formulas for cosines and sines and the fact that $A_{11}+A_{21}+A_{31}=1$ in our case, one finds the expression [38]

$$
\left|\bar{A}_{e e}\right|^{2}=\bar{P}_{e e}=1-\left(P_{21} \sin ^{2} x_{21}+P_{31} \sin ^{2} x_{31}+P_{32} \sin ^{2} x_{32}\right) .
$$

In Eq. (7) we define $P_{21}=4 A_{11} A_{21}, P_{31}=4 A_{11} A_{31}$ and $P_{32}=4 A_{21} A_{31}$. Using $\Delta m_{32}^{2}=\Delta m_{31}^{2}-\Delta m_{21}^{2}$, we rewrite Eq. (77) in a form that is more transparent for discussing the MH question [22], which reads

$$
\begin{aligned}
\bar{P}_{e e}= & 1-\left[\left(P_{21}+\cos \left(2 x_{31}\right) P_{32}\right) \sin ^{2} x_{21}+\left(P_{31}+P_{32}\right) \sin ^{2} x_{31}-\right. \\
& \left.\frac{1}{2} P_{32} \sin \left(2 x_{21}\right) \sin \left(2 x_{31}\right)\right],
\end{aligned}
$$

where the last term is sensitive to the sign of $x_{31}$ and potentially provides a handle on the MH [39]. To the approximation we are working in the NSI formalism, Eqs. (5) and (6) lead to the identifications

$$
\begin{gathered}
A_{11}=c_{13}^{2} c_{12}^{2}-c_{13} \sin \left(2 \theta_{12}\right) c_{23} K_{-}-c_{12}^{2} \sin \left(2 \theta_{13}\right) c_{23} K_{+}, \\
A_{21}=c_{13}^{2} s_{12}^{2}+c_{13} \sin \left(2 \theta_{12}\right) c_{23} K_{-}-s_{12}^{2} \sin \left(2 \theta_{13}\right) c_{23} K_{+}, \\
A_{31}=s_{13}^{2}+\sin \left(2 \theta_{13}\right) c_{23} K_{+},
\end{gathered}
$$

with the conventions for the standard mixing model (SMM) parameters $c_{12} \equiv \cos \left(\theta_{12}\right)$ etc. as defined in 40]. It is evident from Eqs. (9-11) that there are effectively two NSI parameters in the problem, which we have defined in terms of $K_{e \mu}, K_{e \tau}$ and mixing parameters and $\delta$, the standard mixing CP-violating phase, as [41]

$$
\begin{aligned}
& c_{23} K_{+} \equiv\left|K_{e \mu}\right| \cos \left(\delta+\phi_{e \mu}\right) s_{23}+\left|K_{e \tau}\right| \cos \left(\delta+\phi_{e \tau}\right) c_{23}, \\
& c_{23} K_{-} \equiv\left|K_{e \mu}\right| \cos \phi_{e \mu} c_{23}-\left|K_{e \tau}\right| \cos \phi_{e \tau} s_{23} .
\end{aligned}
$$

We will write expressions in terms of these two parameters from now on, factoring out $c_{23}$ for convenience. This makes explicit the reduction of the overall strength of the NSI term by a factor $c_{23} \simeq s_{23} \simeq 1 / \sqrt{ } 2$. The coefficients that appear in Eqs. (77) and (8), namely $P_{21}, P_{31}$ and $P_{32}$, are then given at first order in the $K$ parameters by the expressions

$$
\begin{gathered}
P_{21}=\sin ^{2}\left(2 \theta_{12}\right) c_{13}^{4}+4 c_{13}^{3} \sin \left(2 \theta_{12}\right) \cos \left(2 \theta_{12}\right) c_{23} K_{-}-4 c_{13}^{3} s_{13} \sin ^{2}\left(2 \theta_{12}\right) c_{23} K_{+}, \\
P_{31}=\sin ^{2}\left(2 \theta_{13}\right) c_{12}^{2}-4 s_{13}^{2} c_{13} \sin \left(2 \theta_{12}\right) c_{23} K_{-}+4 c_{12}^{2} \cos \left(2 \theta_{13}\right) \sin \left(2 \theta_{13}\right) c_{23} K_{+},
\end{gathered}
$$

and

$$
P_{32}=\sin ^{2}\left(2 \theta_{13}\right) s_{12}^{2}+4 s_{13}^{2} c_{13} \sin \left(2 \theta_{12}\right) c_{23} K_{-}+4 s_{12}^{2} \cos \left(2 \theta_{13}\right) \sin \left(2 \theta_{13}\right) c_{23} K_{+} .
$$

Taking the parameters $K_{e \mu}$ and $K_{e \tau}$ one at a time, commonly done in setting NSI bounds, costs little generality. $K_{+}$ and $K_{-}$are still independent of each other, because $K_{+}$depends on the real part of $K_{e \mu}$, the imaginary part of $K_{e \mu}$ and the standard mixing phase $\delta$, while $K_{-}$depends only on the real part of $K_{e \mu}$, and similarly for $K_{e \tau}$. The only loss is the possibility of enhancements or cancellations that could allow a larger range of possible values than the quoted one-at-a-time bounds in the literature [10]. 


\section{COMBINING NSI EFFECTS IN SHORT AND MEDIUM BASELINE EXPERIMENTS}

\section{A. NSI and the short baseline reactor neutrino determination of $\sin ^{2}\left(2 \theta_{13}\right)_{\text {eff }}=P_{31}+P_{32}$}

In this case, the term proportional to $\sin ^{2}\left(x_{31}\right)$ in the final form of Eq. (8) dominates the contributions to the short baseline Double Chooz, Daya Bay and RENO experiments. Corrections from $x_{21}=\Delta m_{21}^{2} L / 4 E$ are much smaller than the experimental errors, so to an excellent approximation the transition probability including the NSI effects reads

$$
\bar{P}_{e e}=1-\sin ^{2}\left(\Delta m_{31}^{2} \frac{L}{4 E}\right)\left(P_{31}+P_{32}\right)=1-\sin ^{2}\left(\Delta m_{31}^{2} \frac{L}{4 E}\right)\left[\sin ^{2}\left(2 \theta_{13}\right)+4 \cos \left(2 \theta_{13}\right) \sin \left(2 \theta_{13}\right) c_{23} K_{+}\right],
$$

where the angles $\theta_{13}$ and $\theta_{23}$ are the parameterization angles in the commonly used form of the neutrino mixing matrix [40], and $K_{+}$is defined in Eq. (12). Only the leading order terms in $K$ s are kept in Eq. (17), since the flavor violating coefficients are constrained by experimental searches to be of order 0.05 or less [10]. The fit to data by the Double Chooz, Daya Bay and RENO experiments essentially determine value of the coefficient of $\sin ^{2}\left(\Delta m_{31}^{2} \frac{L}{2 E}\right)$, which in the standard neutrino mixing picture is simply $\sin ^{2}\left(2 \theta_{13}\right)$. When the NSI are included, the measured coefficient of $\sin ^{2}\left(\Delta m_{31}^{2} L / 4 E\right)$ determines the whole expression in brackets in Eq. 17. For example, using the Daya Bay value $\sin ^{2}\left(2 \theta_{13}\right)_{\text {eff }}=0.089 \pm 0.011$ [4] we have

$$
\sin ^{2}\left(2 \theta_{13}\right)_{e f f} \equiv \sin ^{2}\left(2 \theta_{13}\right)+4 \cos \left(2 \theta_{13}\right) \sin \left(2 \theta_{13}\right) c_{23} K_{+}=0.089 \pm 0.011
$$

in our linear approximation [42]. We show in Fig. 1 the curve, with corresponding upper and lower 90\% C.L. uncertainties, of values in the $\left(K_{+}, \sin ^{2}\left(2 \theta_{13}\right)\right)$ plane that satisfy the constraint in the interval $-0.04<K_{+}<0.04$. Not all of the parameter ranges quoted in [10] are covered in this interval if one allows for maximal constructive coherence among the parameters, stretching the interval to roughly $-0.1<K_{+}<0.1$, but it is safely within the region where a linear NSI approximation is reliable.

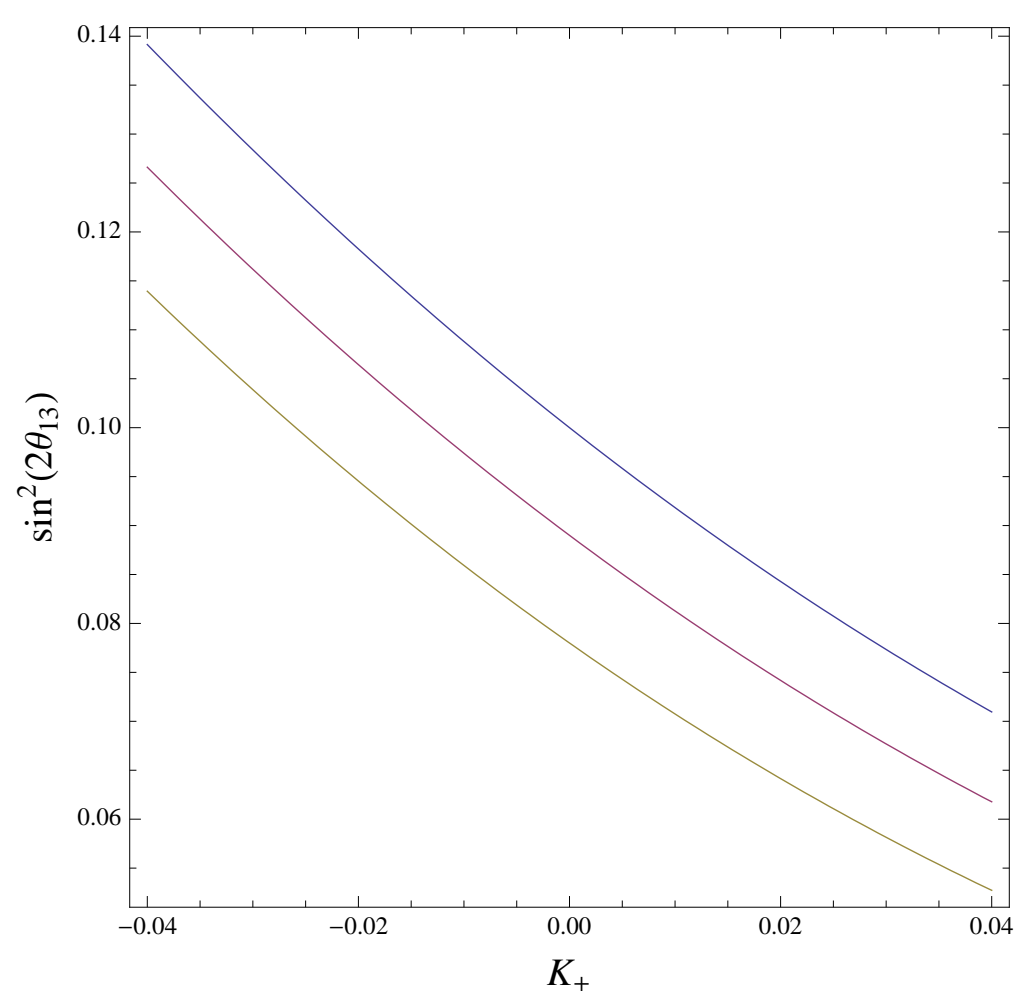

FIG. 1: The Daya Bay result for $\sin ^{2}\left(2 \theta_{13}\right)_{e f f}$ and its one sigma uncertainties [4] are used to constrain the ranges of the parameters $\sin ^{2}\left(2 \theta_{13}\right)$ and the NSI parameter $K_{+}$in Eq. (17).

For this application the effective NSI factor is $\left.K_{+}=\left|K_{e \mu}\right| \cos \left(\phi_{e \mu}+\delta\right)+\left|K_{e \tau}\right| \cos \left(\phi_{e \tau}+\delta\right)\right)$, where we have assumed maximal mixing in the "23" sector, $s_{23}=c_{23}=0.717$ [43]. As is known [9], with the same NSI at source and detector, 
as in our treatment, the NSI effect an overall shift in the coefficient of the dominant $\sin ^{2}\left(x_{31}\right)$ oscillation factor in $\bar{P}_{e e}$. This establishes a strong correlation between the value of $\sin ^{2}\left(2 \theta_{13}\right)$ and the value of the NSI parameter $K_{+}$, a point that will be reiterated in our study of the sensitivity of medium baseline oscillations to the hierarchy question. To resolve $K_{+}$to find evidence for NSI requires a combined analysis with an independent, precision measurement of $\sin ^{2}\left(2 \theta_{13}\right)$, involving different physics from the physics of reactor $\overline{\nu_{e}}$ disappearance. As remarked in Ref. [8], this role is played by accelerator neutrino appearance experiments, a role which can be filled by the ongoing T2K [6] and MINOS [5] experiments, upcoming experiments such as NOvA [44] and future experiments at a neutrino factory [45].

\section{B. Short baseline NSI constraint and medium baseline determination of $P_{21}$ and $P_{32}$}

As the length of the baseline $L$ is increased in the oscillation factor arguments $x_{21}$ and $x_{31}$, the first and third terms in brackets in Eq. (8) play the primary role in determining the fraction of $\bar{\nu}_{e} s$ surviving at distance $L$ from the source. The first term's $P_{21}$ coefficient controls the "slow" oscillations with energy at fixed baseline, while the third term's coefficient, $P_{32}$, controls the "fast" oscillations. Solving Eq. (18) for $\sin ^{2}\left(2 \theta_{13}\right)$ in terms of $K_{+}$, as illustrated in Fig. 1. we can write the $x_{i j}$-independent factors in the probability for $\bar{\nu}_{e}$ disappearance, Eq. (8), completely in terms of the NSI parameters $K_{+}$and $K_{-}$and the standard mixing angles $\theta_{12}$ and $\theta_{23}$. Alternatively, we can eliminate $K_{+}$in terms of $\theta_{13}$ and $\theta_{23}$, as we do in Sec. III.B.2 below. First we take a look at the overall spectral behavior and then turn to a quick survey of the rapid oscillations that contain the information about the MH.

\section{Study of a modeled medium baseline reactor neutrino experiment and the influence of NSI effects}

Here we follow several recent explorations of the prospects for $\mathrm{MH}$ determination within standard model extended by mixed, massive neutrinos. The favored baselines are in the neighborhood of (30-60) $\mathrm{km} \mathrm{[16],} \mathrm{21]} \mathrm{and} \mathrm{[22].} \mathrm{As} \mathrm{in}$ [22] we adopt the neutrino flux model of [46], the gaussian energy resolution smearing model of [22], the cross section of [47] and, the general approach of [22]. The observed neutrino energy distribution for an experiment with a 20 giga watt $(\mathrm{GW})$ thermal power, a detector of 5 kilotons fiducial volume and $12 \%$ weight fraction of free protons with total number of free protons $N_{p}$ and 5 years of exposure time $T$, can be written

$$
\frac{d N}{d E_{\nu}}\left(E_{\nu}\right)=\frac{N_{p} T}{4 \pi L^{2}} \int_{E_{t h}}^{E_{\max }} d E \frac{d N}{d E} \bar{P}_{e e}\left(L, E, K_{+}, K_{-}\right) \sigma_{I B D}(E) G\left(E-E_{\nu}\right)
$$

Here $E_{t h}=m_{n}-m_{p}+m_{e}$, the threshold energy, $\frac{d N}{d E}$ is the rate of neutrino emission from the reactor per $M e V, \bar{P}_{e e}$ is defined in Eqs. (8), (14), (15) and (16), $\sigma_{I B D}$ is the total cross section for inverse beta decay, and $G\left(E-E_{\nu}\right)$ is the gaussian smearing function that takes into account the response of the detector to the deposited energy.

$$
G\left(E-E^{\prime}, \sigma_{E}(a, b)\right)=\frac{1}{\sqrt{2 \pi} \sigma_{E}(a, b)} \exp \left[-\frac{\left(E-E^{\prime}\right)^{2}}{2\left(\sigma_{E}(a, b)\right)^{2}}\right] .
$$

The uncertainty $\sigma_{E}(a, b)$ in the energy $E$ is parameterized in terms of a statistical parameter $a$ and a systematic parameter $b$ as

$$
\sigma_{E}(a, b)=E\left[\frac{a^{2}}{E}+b^{2}\right]^{1 / 2}
$$

where energy is in $\mathrm{MeV}$ and the first term under the square root represents the statistical uncertainty in the energy deposited in the detector and the second represents the systematic energy scale uncertainty.

Computing the energy spectrum expected in the model described, we show the results at a baseline of $50 \mathrm{~km}$ with perfect detector response (no smearing) in Fig. 2. Table \ displays values and their uncertainties for the relevant input parameters.

The left plot shows variation of the spectrum with central values of all input parameters and different NSI parameter choices. NH curves are shown in blue, IH in red. When $x_{21}=\pi / 2$ at $E_{\nu} \sim 3 G e V$, the maximum and zero in the factors $\sin ^{2}\left(x_{21}\right)$ and $\sin \left(2 x_{21}\right)$, respectively, is evident in the figure. The right plot shows the variation of the spectrum with no NSI and, from top to bottom, $\sin ^{2}\left(2 \theta_{12}\right)$ at its central value minus $1 \sigma$, its central value and its central value plus $1 \sigma$. The middle curves on the left and right are the same, of course, and are included for reference [48]. As we 


\begin{tabular}{|l|l|l|l||}
\hline $\sin ^{2}\left(2 \theta_{12}\right)$ & $\sin ^{2}\left(2 \theta_{13}\right)$ & $\Delta m_{21}^{2}$ & $\Delta m_{31}^{2}$ \\
\hline \hline $0.857 \pm 0.024^{*}[27, \underline{40]}$ & $0.089 \pm 0.011^{*}[2]$ & $(7.50 \pm 0.20) 10^{-5 *}[40]$ & $(2.32 \pm 0.10) 10^{-3 *}[40]$ \\
\hline $0.869_{-0.037}^{+0.031}[49]$ & & & \\
\hline $0.845_{-0.071}^{+0.066}[27]$ & & & \\
\hline
\end{tabular}

TABLE I: Values of relevant input parameters. The values marked with asterisks are the ones used in the numerical work to illustrate the ideas and make estimates.

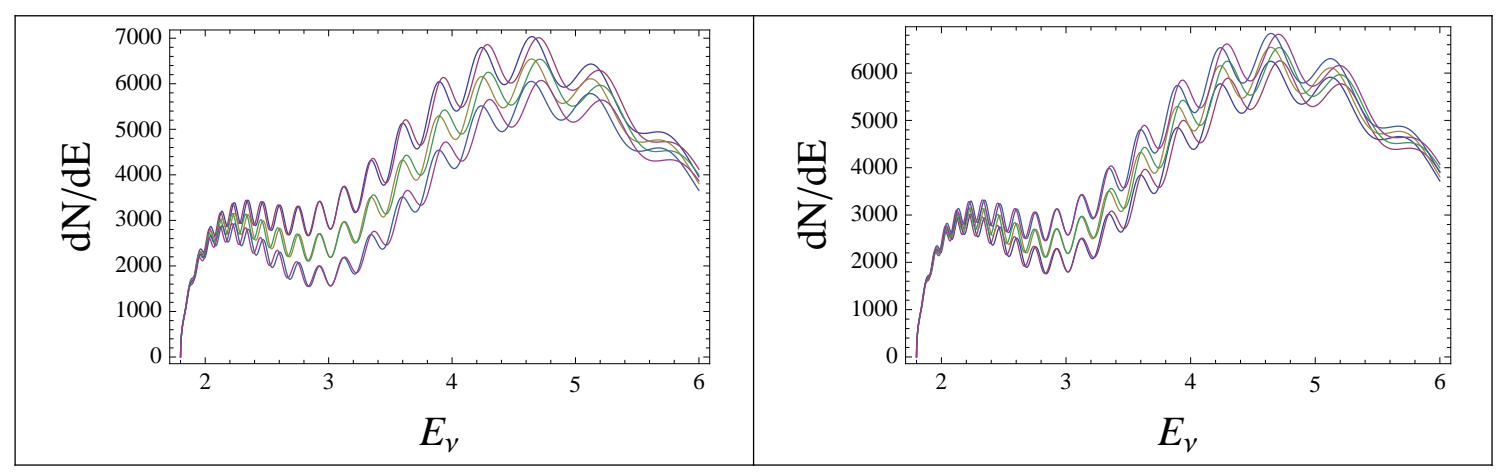

FIG. 2: The predicted rates of events with a $50 \mathrm{~km}$ baseline for the medium baseline set up described in the text with perfect detector energy response, $\mathrm{a}=\mathrm{b}=0 . E_{\nu}$ is in $\mathrm{MeV}$ and $\mathrm{dN} / \mathrm{d} E_{\nu}$ is in inverse $\mathrm{MeV}$. The left plot shows variation of the spectrum with central values of all parameters and different NSI choices. From top to bottom: $K_{+}=-0.04=K_{-}$, where $P_{32}$ is minimal, $K_{+}=0=K_{-}$, no NSI, and $K_{+}=-0.04$ and $K_{-}=+0.04$, where $P_{32}$ is maximal. NH curves are shown in blue, IH in red. The right plot shows the variation of the spectrum with no NSI and $\sin ^{2}\left(2 \theta_{12}\right)$ ranging within its $1 \sigma$ uncertainty. The middle curves on the left and right are the same.

discuss in the following subsection, the ordering is expected, since the survival probability is smallest and the rates of detection are smallest when the "oscillation term", which represents transitions into other flavors, is largest within a given range of parameter space. The parameter choice that makes the $\mathrm{MH}$-sensitive term, $P_{32}$, largest also makes the overall oscillation term in Eq. (17) take its largest value and makes the survival rate the smallest. For illustration we have chosen NSI magnitudes that are about as large as allowed by the current experimental constraints on NSI parameters when taken one at a time.

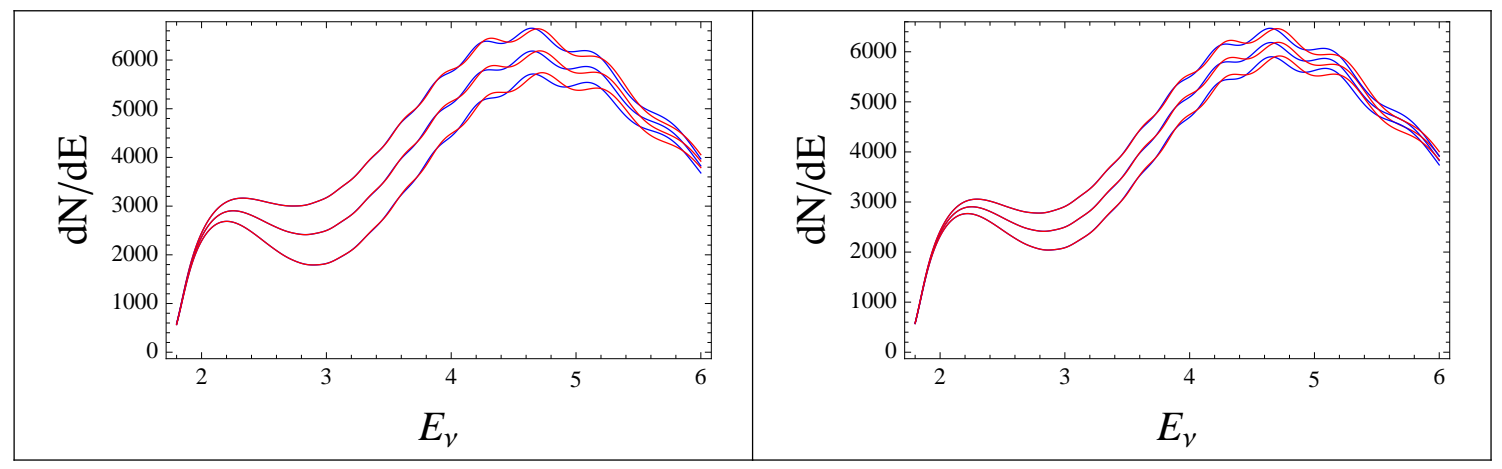

FIG. 3: The predicted rates of events with a $50 \mathrm{~km}$ baseline for the medium baseline set up described in the text with $a=6 \%$ and $b=0$ uncertainties in the detector energy response. $E_{\nu}$ is in $\mathrm{MeV}$ and $d N / d E_{\nu}$ is in inverse MeV. The left plot shows variation of the spectrum with central values of all input parameters and different NSI choices : top to bottom are $K_{+}=K_{-}$ $=-0.04, K_{+}=K_{-}=0, K_{+}=-0.04$ and $K_{-}=+0.04$. NH curves are shown in blue, IH in red. The right plot shows the variation of the spectrum with no NSI and $\sin ^{2}\left(2 \theta_{12}\right)$ ranging within its $1 \sigma$ uncertainty. The middle curves on the left and right are the same. 
In Fig. 3 we show the same cases but with energy uncertainty fractions $a=6 \%$ and $b=0$. Within this range of NSI possibilities, it is clear that the effects on the spectrum can be large and mimic those of the uncertainty in the value of $\sin ^{2}\left(2 \theta_{12}\right)$. If the value of $\sin ^{2}\left(2 \theta_{12}\right)$ could be determined with high precision from solar neutrino data, for example, then a combined analysis with the medium baseline spectrum could disentangle the input uncertainties and the NSI andprovide a sensitive probe of the NSI parameters $K_{-}$. In Sec. IV. we show in another way that within the current uncertainties in $\sin ^{2}\left(2 \theta_{12}\right)$, there is a degeneracy between $K_{-}$and $\sin ^{2}\left(2 \theta_{12}\right)$ in interpreting medium baseline data, as one can see in the expression for $P_{21}$ in Eq. 14, the dominant coefficient of $\sin ^{2} x_{21}$. Changes in the value of $\sin ^{2}\left(2 \theta_{12}\right)$ can be compensated by changes in $K_{-}$, for example. In terms of Figs. 2 and 3 . one finds that the left and right plots are essentially identical if one choses $\left|K_{+}\right|=\left|K_{-}\right|=0.024$ and the central value of $\sin ^{2}\left(2 \theta_{12}\right)$ for the left plot. We will return to this issue in Sec. IV.

In Fig. 4, we show the envelopes of all the spectra possible within the parameter ranges for $\left|K_{+}\right|,\left|K_{-}\right|$and $\sin ^{2}\left(2 \theta_{12}\right)$. It is clear that there is a wide range of possible spectra beyond that expected from the uncertainty in the input values of the mixing angles when the NSI effects are included.

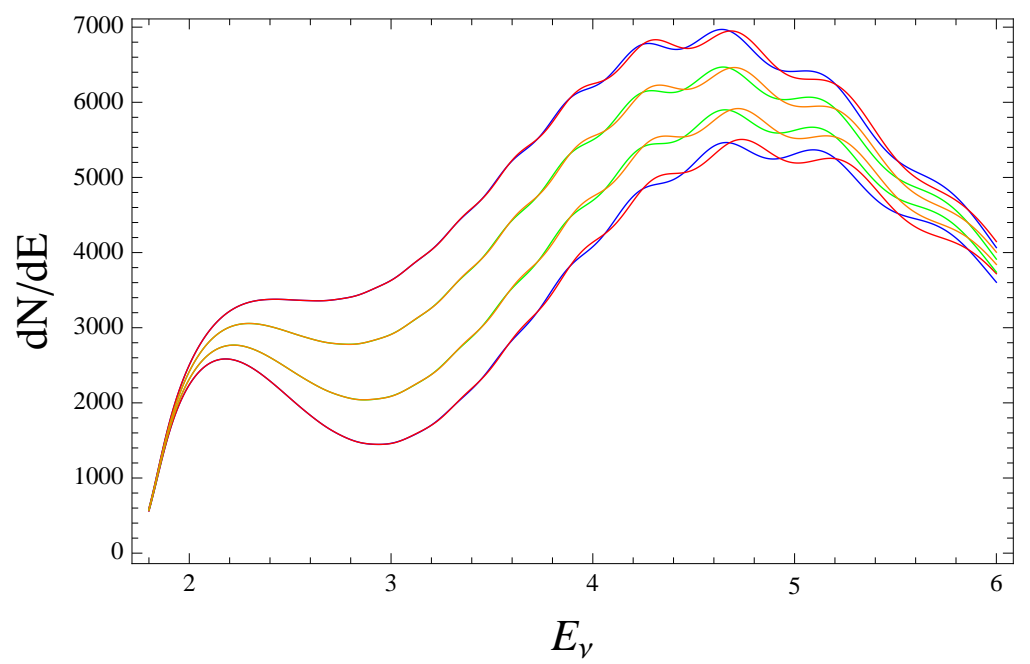

FIG. 4: Allowing our full range values for the NSI parameters $|K+|$ and $\left|K_{-}\right|$and the mixing angle $\theta_{12}$, we find the outer envelope of spectral curves indicated in the figure. The envelope of curves expected just from the $1 \sigma$ variation of $\theta_{12}$ is shown inside the full envelope. $E_{\nu}$ is in $\mathrm{MeV}$ and $\mathrm{dN} / \mathrm{d} E_{\nu}$ is in inverse $\mathrm{MeV}$.

The differences in the overall rates are much larger in Figs. 2 and 3 than the purely statistical fluctuations in counting estimated by the square root of the event numbers, even with the energy smearing. This fact is behind the expectation of highly improved precision in determination of the coefficient of $\sin ^{2} x_{21}$ [22], $P_{21}, \sin ^{2}\left(2 \theta_{12}\right)$ in the SMM. The primary effect of the smearing is to reduce the distinction between NH and IH expected spectra, as studied in 22]. We show this in a different, more direct way in Fig. 5.

Taking the difference between the NH rate prediction and the IH prediction gives a clearer picture of the distinction between the two possibilities. In Fig. 5 the left panel shows the difference between the NH and IH predictions as a function of energy with a baseline of $50 \mathrm{~km}$ when the resolution is perfect. For this ideal situation, the distinction between the $\mathrm{NH}$ and $\mathrm{IH}$ predictions is clear in the maximal and no NSI cases but marginal in the minimal case. When the uncertainty in the energy resolution is $6 \%$ and the energy scale error is 0 , the distinctions are marginal at best in the maximal case and insufficient in the other cases. In 22] this case was made in detail for the SMM, where the authors concluded that a resolution of better than $3 \%$ would be necessary at the optimal baseline to resolve the hierarchy in a reasonable time. In the right panel in Fig. [5] we show the plot of the difference between $\mathrm{NH}$ and IH rates as a function of energy at $50 \mathrm{~km}$ baseline with a $6 \%$ statistical energy resolution uncertainty and no statistical uncertainty as modeled by the gaussian smearing function. As shown in the figure, the NSI can cause a shift of up to $12 \%$ in the sensitivity of the spectrum to the MH. With no NSI but allowing for a $1 \sigma$ variation in the value of $\sin ^{2}\left(2 \theta_{13}\right)$, the results look nearly the same as shown in Fig. 5, reflecting the $12 \%$ uncertainty in $\sin ^{2}\left(2 \theta_{13}\right)$ eff . When the anticipated $4 \%$ goal for the uncertainty in $\sin ^{2}\left(2 \theta_{13}\right)_{\text {eff }}$ is reached, the sensitivity to NSI effects will be correspondingly enhanced by a factor 3 . The uncertainty in $\sin ^{2}\left(2 \theta_{12}\right)$ allows a shift of about $3 \%$. 


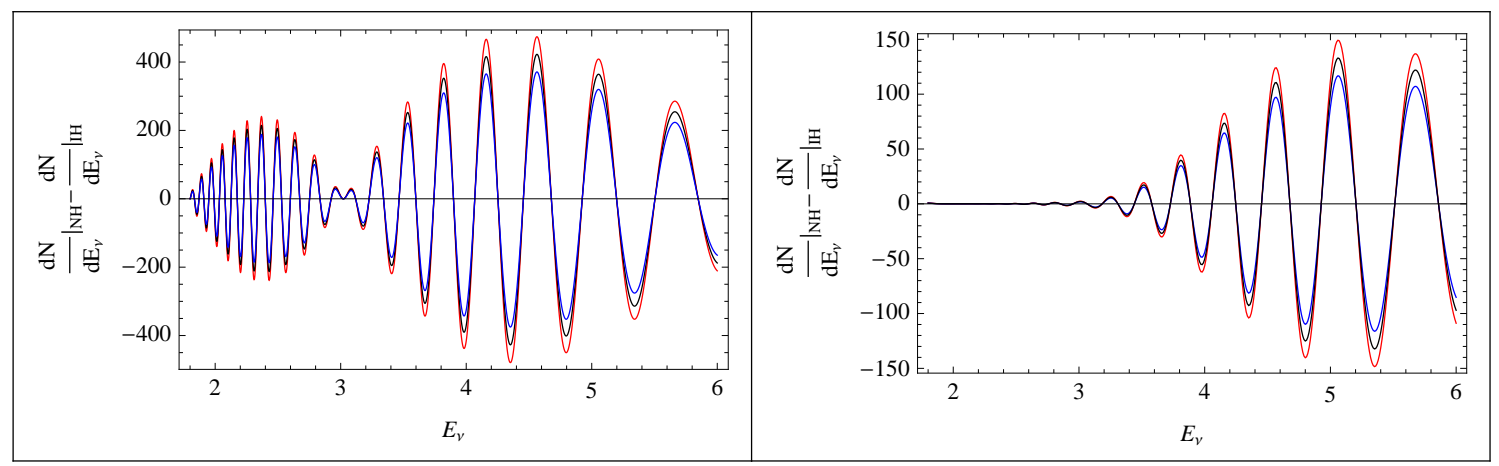

FIG. 5: The predicted difference between rates of $\mathrm{NH}$ and $\mathrm{IH}$ events as a function of energy with a $50 \mathrm{~km}$ baseline for the medium baseline set up described in the text. $E_{\nu}$ is in $\mathrm{MeV}$ and $d N / d E_{\nu}$ is in inverse MeV. The left panel shows the result with no uncertainty in the detector energy resolution and energy scale and the right shows the result with $6 \%$ uncertainty in the detector energy resolution. From top to bottom: $K_{+}=-0.04$ and $K_{-}=+0.04$, where $P_{32}$ is maximal, $K_{+}=0=K_{-}$, no NSI, and $K_{+}=-0.04=K_{-}$, where $P_{32}$ is minimal. Input parameters are set at their central values. Note the difference in scale between the two plots.

\section{The influence of the NSI parameters $K_{+}$and $K_{-}$on the factors $P_{21}$ and $P_{32}$ in the disappearance probability}

The coefficient that controls the scale of long oscillation length behavior of the disappearance probability is $P_{21}$, which has the signature feature that it is independent of the NSI parameter $K_{+}$to a high degree of accuracy. Over the range $-0.04 \leq K_{+} \leq 0.04$, the change in $P_{21}$ for any choice of $K_{-}$in the same range is at most $0.1 \%$, typically 100 times smaller than the change due to $K_{-}$over this range. To see how this happens, it is helpful to use Eq. (18) to eliminate $K_{+}$and rewrite Eq. (14) in the form

$$
P_{21}=\sin ^{2}\left(2 \theta_{12}\right) c_{13}^{4}\left(1-4 \tan \theta_{13} \frac{\left(\sin ^{2}\left(2 \theta_{13}\right)_{e f f}-\sin ^{2}\left(2 \theta_{13}\right)\right)}{\sin \left(2 \theta_{13}\right) \cos \left(2 \theta_{13}\right)}\right)+4 c_{13}^{3} \sin \left(2 \theta_{12}\right) \cos \left(2 \theta_{12}\right) c_{23} K_{-} .
$$

The factor $c_{13}^{4}$ multiplying $\sin ^{2}\left(2 \theta_{12}\right)$ in the first term increases monotonically as $\sin ^{2}\left(2 \theta_{13}\right)$ decreases from its maximum value to its minimum value, while the factor in the large parentheses decreases monotonically. The two effects compensate each other to high accuracy. The whole first term is of order 1 . The coefficient of $K_{-}$in the second term increases monotonically by $2 \%$ over the same range. The magnitude of the coefficient is close to 1 , so the size of the second term relative to the first is determined by $K_{-}$, which ranges between -0.04 and +0.04 . The net effect is that $P_{21}$ varies by at most $0.1 \%$ with $-0.04<K_{+}<+0.04$ for any chosen values of $K_{-}$and the input values of mixing angles within their uncertainties.

The dependence of the MH-sensitive coefficient $P_{32}$ on the NSI parameters can be substantial [50]. The value of $P_{32}$ largely controls the possibility of determining the sign of the last term in Eq. (8) and, therefore, whether $m_{3}>m_{1}(\mathrm{NH})$ or $m_{1}>m_{3}(\mathrm{IH})$. The effects of new flavor violating interactions on $P_{32}$ increase as $K_{+}$decreases, which corresponds to increasing values of $\sin ^{2}\left(2 \theta_{13}\right)$, shown in Fig. 1. The smallest values of $P_{32}$ occur at the smallest values of $K_{+}$and $K_{-}$. For example, at $(-0.05,-0.05), \frac{1}{2} P_{32}=0.0115$, while at $(-0.05,+0.05), \frac{1}{2} P_{32}=0.0165$. To understand the dependence of $P_{32}$ on the $\left(K_{+}, K_{-}\right)$parameters, again it is helpful to use the definition of $\sin ^{2}\left(2 \theta_{13}\right)_{\text {eff }}$ in Eq. (18) to rewrite $P_{32}$ in an equivalent form as

$$
P_{32}=s_{12}^{2} \sin ^{2}\left(2 \theta_{13}\right)_{e f f}+4 s_{13}^{2} c_{13} \sin \left(2 \theta_{12}\right) c_{23} K_{-},
$$

in which $P_{32}$ has no explicit dependence on $K_{+}$. Through the constraint Eq. (18), $P_{32}$ has implicit dependence on $K_{+}$via the factor $s_{13}^{2} c_{13}$ in the coefficient of $K_{-}$. For fixed $K_{+}$, the value of $P_{32}$ is linearly dependent on $K_{-}$ with a positive coefficient. Because of the constraint imposed by Eq. (18), $\sin \left(\theta_{13}\right)$ decreases as $K_{+}$increases, which decreases the coefficient of $K_{-}$, and conversely when $K_{+}$decreases. In short, $P_{32}$ increases as $K_{+}$grows when $K_{-}<0$ and decreases as $K_{+}$grows when $K_{-}>0$, though the dependence is weak compared to the dependence on $K_{-}$.

The upshot is that our parameterization in terms of $K_{-}$and $K_{+}$has the good feature that we can choose to write the coefficients $P_{21}$ and $P_{32}$ with only $K_{-}$involved explicitly in the NSI effects on overall spectrum shape and, as we argue below, in the sensitivity, or not, of the MH to NSI. The parameter $K_{+}$plays an indirect role through its relationship with the measured $\sin ^{2}\left(2 \theta_{13}\right)_{\text {eff }}$ and the mixing angle $\theta_{13}$, Eq. (18). The weak dependence on $\sin ^{2}\left(2 \theta_{13}\right)$ is transparent and makes the minor role of $K_{+}$easier to understand. 
A general feature of $P_{32}$, the "contrast coefficient" between the $\mathrm{NH}$ and $\mathrm{IH}$ rate values, is that for all positive values of $K_{-}$the differences between $\mathrm{NH}$ and $\mathrm{IH}$ rates are larger than those for standard mixing and the overall rates are smaller, while the opposite is true for all negative values of $K_{-}$. When $K_{-}=0$, there is a degeneracy that is almost complete; given an input value of $\sin ^{2}\left(2 \theta_{23}\right)_{e f f}$, Eq. (18), $P_{32}^{N S I}=P_{32}^{S M M}$ and $P_{31}^{N S I}=P_{31}^{S M M}$, and $P_{21}^{N S I}-P_{21}^{S M M} \ll 1$ for all values of $K_{+}$within current experimental bounds. Only for values of $K_{-} \neq 0$ can the presence of NSI be detected with any confidence. How large must $K_{-}$be for its detection to be possible? We address this question in the following section.

\section{STATISTICAL SENSITIVITY OF THE SPECTRUM TO THE NSI PARAMETERS}

The discussion in Sec. III.B.2 laid out the main features of the NSI parameters' influence on $P_{21}$ and $P_{32}$, where the first controls the long oscillation length behavior of the spectrum and the second controls the contrast between the $\mathrm{NH}$ and $\mathrm{IH}$ short oscillation length behaviors of the spectrum. The examples shown in Figs. 2 through 5 are chosen with values of the NSI parameters suggested by the individual parameter bounds [10] in order to make the effects visually clear. In this section we make the pictures more quantitative by using simple $\chi^{2}$ estimates of the deviation from the SMM when NSI effects are included, while illustrating the interplay with the input parameter uncertainties by letting key inputs range over their one standard deviation values.

\section{A. Fitting SMM "data" with NSI fit function and $\sin ^{2}\left(2 \theta_{12}\right)$ "pull"}

As an estimator, we adopt the "no NSI", or SMM, values for the spectrum as data and the square root of the number of predicted events as the statistical uncertainty. Using the same points spaced at intervals of $0.01 \mathrm{MeV}$ from which Figs. 2 through 5 were made, but with the smearing parameters chosen as $a=0.03 \%$ and $b=0$, we compute

$$
\chi^{2}=\Sigma_{i}\left(\frac{{\frac{d N}{d E_{\nu}}}^{N S I}-{\frac{d N}{d E_{\nu}}}^{S M M}}{\sqrt{ }{\frac{d N}{d E_{\nu}}}^{S M M}}\right)_{i}^{2}\left(\Delta E_{\nu}\right)_{i} .
$$

Input parameters are fixed at central values for $\frac{d N^{S M M}}{d E_{\nu}}$, while one or more are allowed to vary over their $1 \sigma$ ranges for $\frac{d N^{N S I}}{d E_{\nu}}$. Because $\chi^{2}=0$, its minimum, when NSI parameters are zero and the input parameters are at their central value, the value of $\Delta \chi^{2} \equiv \chi^{2}-\chi_{\min }^{2}$ is the same as $\chi^{2}$, up to a pull "penalty" term that contributes to the $\chi^{2}$ value for each input parameter that is varied away from its central value.

Since the spectrum shape at a given medium baseline value is controlled by $\Delta m_{21}^{2}$, and $\sin ^{2}\left(2 \theta_{12}\right)$ determines the scale of the oscillations, we do a statistical analysis allowing the latter to vary away from its central value of $\left\langle\sin ^{2}\left(2 \theta_{12}\right)\right\rangle=0.857$ in the NSI fit function, while the SMM "data" has its input parameters fixed at their central values. There is an implicit, additive "pull" term $\left(\left(\sin ^{2}\left(2 \theta_{12}\right)-\left\langle\sin ^{2}\left(2 \theta_{12}\right)\right\rangle\right) / \sigma_{12}\right)^{2}$ in Eq. (24). In practice we search for a minimum in $\chi^{2}$ with $K_{-}$and $K_{+}$for a range of values of $\sin ^{2}\left(2 \theta_{12}\right)$, chosen simply for illustration to be within its quoted $1 \sigma$ uncertainty range, $\sigma_{12}=0.024$. Graphically, we show the example of the results when $K_{+}=0$ and $\sin ^{2}\left(2 \theta_{12}\right)=0.857$ in Fig. 6 and the example when $\sin ^{2}\left(2 \theta_{12}\right)=\left\langle\sin ^{2}\left(2 \theta_{12}\right)\right\rangle+\sigma_{12}=0.881$ in Fig. 7 . The landscapes of $\chi^{2}$ values in the $\left(K_{+}, K_{-}\right)$plane are all similar to that shown in Fig. 8 for the case where $\sin ^{2}\left(2 \theta_{12}\right)=0.857$, with a near degeneracy in $K_{+}$along fixed $K_{-}$values. Again, since $P_{21}$ is independent of $K_{+}$to an excellent approximation, the dependence of $\chi^{2}$, Eq. (24) on the value of $K_{+}$is negligible and the results are insensitive to the choice of energy uncertainty parameters. In Fig. [6 overlaid is the plot of $\chi^{2}$ vs. $\delta \sin ^{2}\left(2 \theta_{12}\right)$, the deviation from the central value, with $K_{+}=K_{-}=0$. The two plots are degenerate to a fraction of a percent!

Modulo the $\chi^{2}=1$ penalty, Fig. 7 shows that the fit is essentially degenerate with the fit with $\chi^{2}=0$ at $\sin ^{2}\left(2 \theta_{12}\right)=0.857$ and with values of $K_{-}$consistent with current bounds on NSI and $\sin ^{2}\left(2 \theta_{12}\right)$ at the upper end of its current $1 \sigma$ range. As one explores other values of $\sin ^{2}\left(2 \theta_{12}\right)$, the minimum of $\chi^{2}$ moves to the left for larger values, or to the right and on into positive values of $K_{-}$for smaller values of $\sin ^{2}\left(2 \theta_{12}\right)$. This feature is a result of the interplay between the two terms in Eq. (22), where increases in $\sin ^{2}\left(2 \theta_{12}\right)$ must be compensated by decreases in $K_{-}$to keep the minimum $\chi^{2}$ at zero. Table I gives the location in the $K_{-}$variable and values of the corresponding minimum of $\chi^{2}$ for selected values of $\sin ^{2}\left(2 \theta_{12}\right)$ in the range $0.857 \pm 0.024$. Parameter values are $K_{+}=0, a=0.03$ and $b=0$, and $\sin ^{2}\left(2 \theta_{13}\right)_{\text {eff }}=0.089$, but fits have very little sensitivity to these choices. The "penalty" term added to values $\chi^{2}$ gives the weight against values of $\sin ^{2}\left(2 \theta_{12}\right)$ away from the central value. This weighting breaks the degeneracy between the variables $\sin ^{2}\left(2 \theta_{12}\right)$ and $K_{-}$. It guarantees that values of $K_{-}$"hiding behind" $\sin ^{2}\left(2 \theta_{12}\right)$ 


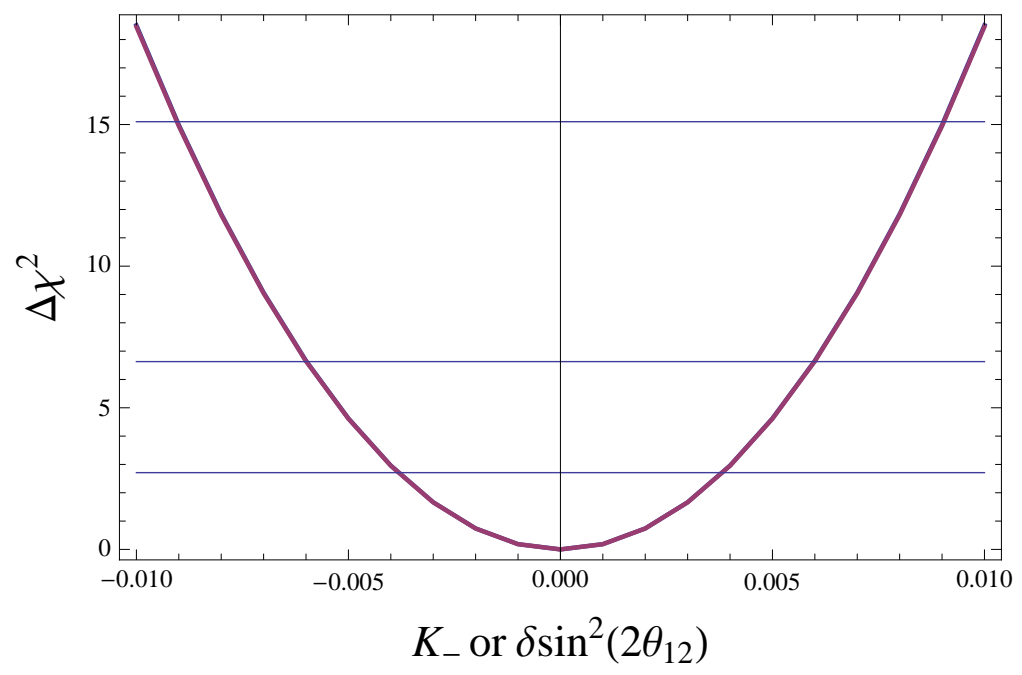

FIG. 6: For $K_{+}$and $K_{-}$ranging between -0.01 and +0.01 , we show the boundaries of the 90\% C.L., the $99 \%$ C.L. and the 99.99\%C.L. projected on a constant $K_{+}$plane. The high degree of degeneracy along the lines of fixed $K_{-}$, see Fig. 8 shows that $\Delta \chi^{2}$ is independent of $K_{+}$to high accuracy, and the problem reduces to 1 parameter. The energy smearing and systematic parameters chosen for the plots are $a=0.03$ and $b=0$, respectively, but the changes in the plot are negligible when any values $a \leq 0.06$ and $b \leq 0.01$ are used.

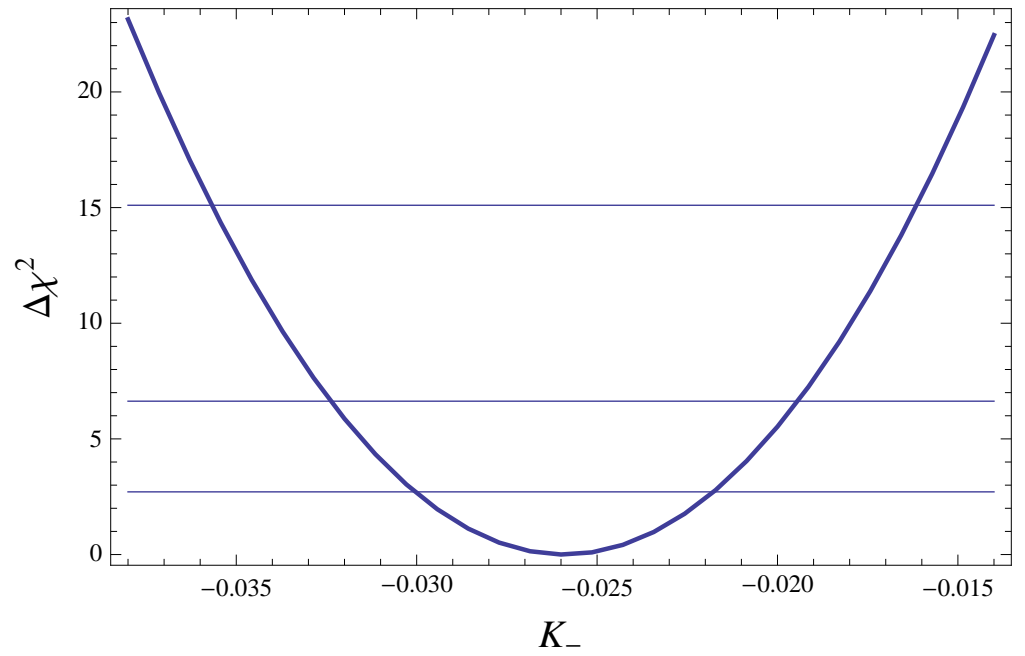

FIG. 7: The various C.L. limits for a fit to SMM with NSI and $\sin ^{2}\left(2 \theta_{12}\right)=0.881$. The other input parameters are set at their current central values and the energy uncertainty parameters are $a=0.03$ and $b=0$, while $K_{+}=0$ for the plot. The results are not sensitive to these choices. For clarity of display, the additive $\chi^{2}=1$ penalty is not included in the plot.

\begin{tabular}{|l|l|c|c|c|c|c|c|}
\hline $\sin ^{2}\left(2 \theta_{12}\right)$ & 0.881 & 0.873 & 0.865 & 0.857 & 0.849 & 0.841 & 0.833 \\
\hline \hline$\left.K_{-}\right|_{\text {min }}$ & -0.0259 & -0.0168 & -0.0082 & 0.0 & 0.0078 & 0.0153 & 0.0225 \\
\hline$\chi_{\text {min }}^{2}$ & $210^{-4}$ & $310^{-5}$ & $510^{-5}$ & 0.0 & $210^{-5}$ & $210^{-5}$ & $810^{-5}$ \\
\hline "penalty" & 1.0 & 0.44 & 0.11 & 0.0 & 0.11 & 0.44 & 1.0 \\
\hline
\end{tabular}

TABLE II: At selected values of $\sin ^{2}\left(2 \theta_{12}\right)$ within its $1 \sigma$ range, we list the values of the variable $K_{-}$at the minimum $\chi^{2}$, with its value, for the test of the NSI NH fit to SMM "data" with central values for all its input parameters. The bottom row lists the value of the additive "penalty" term $\left(\left(\sin ^{2}\left(2 \theta_{12}\right)-0.857\right) / 0.024\right)^{2}$ understood in the $\chi^{2}$ definition, Eq. (24). Energy detection statistical and systematic error parameters are chosen at $a=0.03$ and $b=0$. 


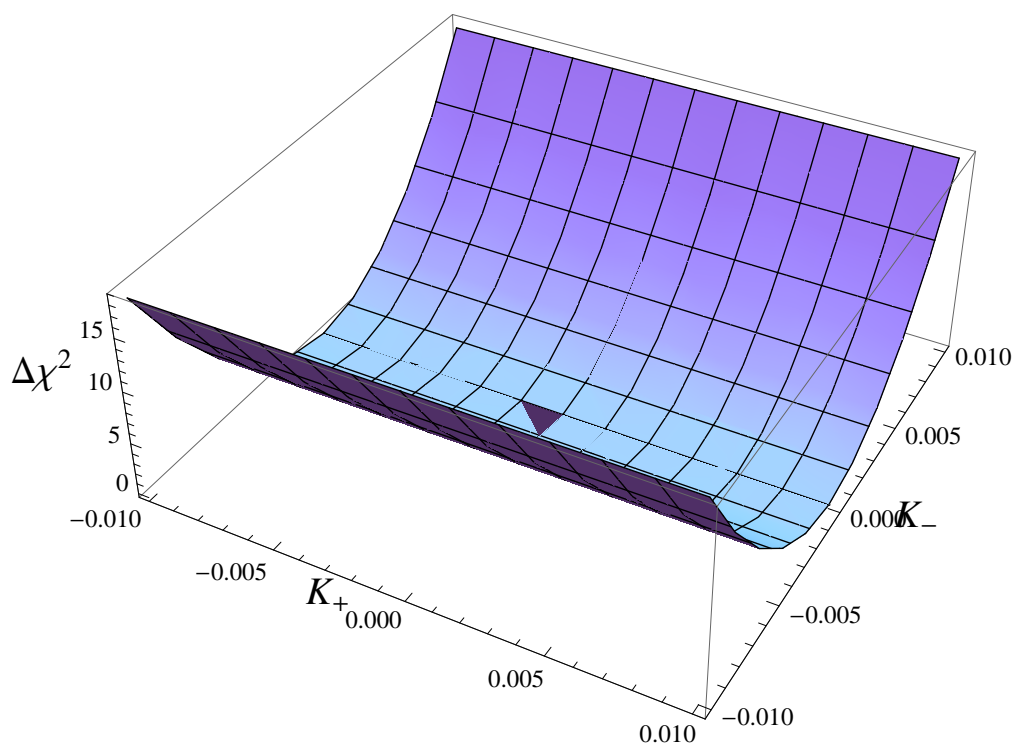

FIG. 8: For $K_{+}$and $K_{-}$ranging between -0.01 and +0.01 and the special case where all input parameters are set to their central values for $\frac{d N^{N S I}}{d E_{\nu}}$, we show the values of $\chi^{2}$ as computed in Eq. (24). The degeneracy along the line $K_{-}=0$ is obvious.

become less and less likely as deviations from the central value grow. The size of values of $K_{-}$are thereby limited in the same measure as the digressions of $\sin ^{2}\left(2 \theta_{12}\right)$ away from its central input value.

This exercise demonstrates that a confusion effect arises in the analysis of medium baseline experiments. A perfectly good fit to data by the SMM with preferred input parameters can be mimicked by a NSI fitting model with a choice of $\sin ^{2}\left(2 \theta_{12}\right)$ that is different from the SMM "data" choice, making the procedure of constraining input parameters by using the reactor neutrino data alone seriously model dependent. However, the smaller the uncertainties in the input parameter $\sin ^{2}\left(2 \theta_{12}\right)$ that are determined from other, independent experiments with different physics at the detector and combined with the medium baseline reactor analysis, the smaller the allowed range of values of NSI parameters from zero. The combined data becomes more sensitive to the presence of NSI. In the example above, values of $\left|K_{-}\right| \geq 0.026$ are ruled out at $1 \sigma$. With the conventional one degree of freedom connection between confidence level (C.L.) and $\chi^{2},\left|K_{-}\right| \geq 0.04$ is ruled out at 90\% C.L., which is essentially the same as the currently available bounds. Improvements in uncertainty in $\sin ^{2}\left(2 \theta_{12}\right)$ in future experiments like $S N O+[51]$ and Hyper-Kamiokande [5]] will improve the sensitivity of medium baseline reactor experiments to the $K_{-}$parameter by the same factor as the improvement in input parameter precision.

As pointed out earlier, whether the distinction between the $\mathrm{NH}$ and $\mathrm{IH}$ cases is stronger or weaker when NSI are present than it is in the SMM case depends upon the sign of $K_{-}$. This gives another handle on the detailed dependence on $K_{-}$and $K_{+}$, and we investigate the sensitivity of the $\mathrm{MH}$ determination from medium baseline data to these parameters.

\section{NH VS. IH AS A FUNCTION OF THE NSI PARAMETERS $K_{+}$AND $K_{-}$}

Our measure of the distinction between the spectrum expected in the NH mass splitting case vs. the spectrum in the $\mathrm{IH}$ case is in the form of a $\chi^{2}$ that assumes data given by one case and the fit attempted with the other 22]. Statistical and systematic uncertainties, as well as uncertainties of the input parameters, will blur the contrast between the two MH possibilities. Assuming that the IH is the data, for example, we write the corresponding $\chi^{2}$ as

$$
\Delta \chi_{M H}^{2}=\Sigma_{i=1}^{N}\left(\frac{{\frac{d N}{d E_{\nu}}}^{N H}-{\frac{d N}{d E_{\nu}}}^{I H}}{\sqrt{\frac{d N}{d E_{\nu}}}}{ }^{I H}\right)_{i}^{2}\left(\Delta E_{\nu}\right)_{i},
$$

where $\left(\Delta E_{\nu}\right)_{i}$ is the width of the $i^{t h}$ energy bin. It is understood that the NH and IH rates depend on the NSI parameters $K_{+}$and $K_{-}$(which can be taken as zero to regain the standard mixing result) as well as the standard 3-neutrino mixing angles $\theta_{i j}$, mass-squared differences $\Delta m_{21}^{2}$ and $\Delta m_{31}^{2}$ and the baseline $L$. Again, the summation is 
over the "data bins", which we take every $0.01 \mathrm{MeV}$ between 1.8 and $8 \mathrm{MeV}$. Using Eq. (25), we look at the effects of varying the NSI parameters, effectively marginalizing only over the range of input parameter values, $\Delta m_{31}^{2}$, which has the only significant impact on the value of $\chi^{2}$, as pointed out in 22].

In Fig. 9 we show the $\Delta \chi_{M H}^{2}$ landscape produced by Eq. [25] for the value $\Delta m_{31}^{2}=2.343 \times 10^{-3}$, where $\Delta \chi_{M H}^{2}$ takes its minimum value when NSI are not present. The figure is normalized by $\Delta \chi_{M H}^{2}$ for $K_{-}=K_{+}=0$, the SMM case. The baseline is $50 \mathrm{~km}$, central values are chosen for all input parameters except $\Delta m_{31}^{2}$, and the energy uncertainty parameters are taken for illustration to be $a=0.03$ and $b=0$, which are among the values explored in [22]. The study presented here is the most straightforward generalization to NSI of their analysis. As explained in Sec. III.B.2, for any

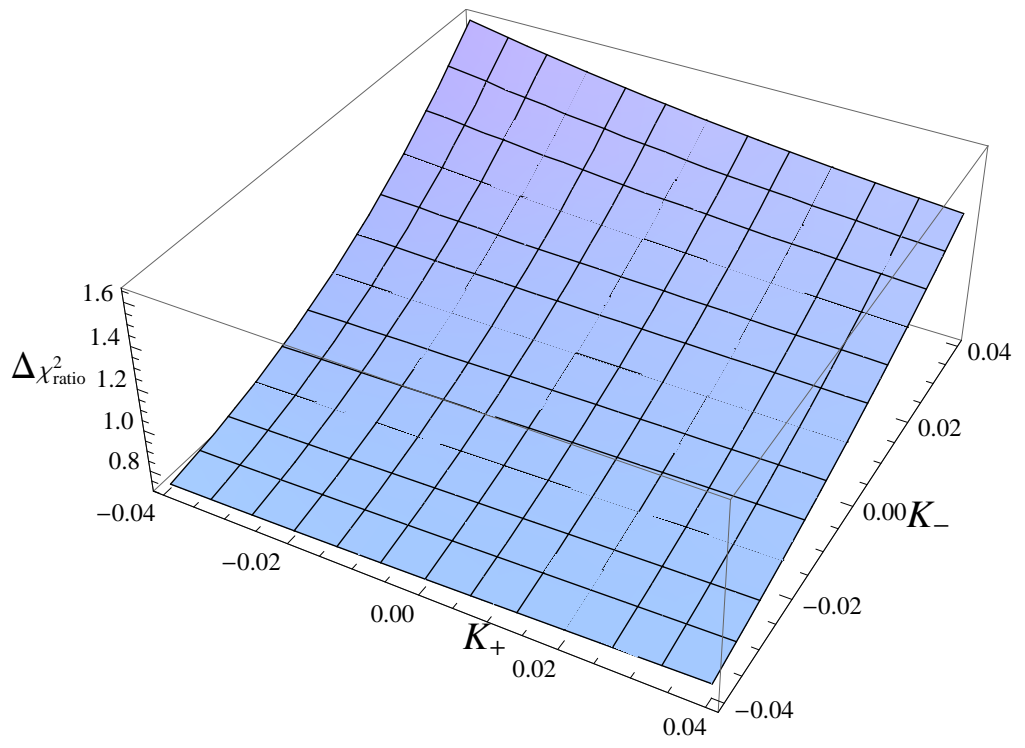

FIG. 9: For $K_{+}$and $K_{-}$ranging between -0.04 and +0.04 , we show the values of $\Delta \chi_{M H}^{2}\left(K_{+}, K_{-}\right) / \Delta \chi_{M H}^{2}(0,0)$ at the value of $\Delta m_{31}^{2}$ where $\Delta \chi_{M H}^{2}$ is a minimum. The case where IH is assumed to be the "data", as computed in Eq.(25), is used for the figure.

value of $\Delta m_{31}^{2}$ the smallest difference between $\mathrm{NH}$ and IH should occur where $P_{32}$ is smallest and the largest difference should occur when $P_{32}$ is largest. This is qualitatively reflected in the behavior shown in Fig. 9. The features of the plots as they depend on $K_{+}$and $K_{-}$are explained in detail in Sec. III.B.2. For any value of $K_{+}$, the sensitivity to the MH as measured by $\Delta \chi^{2}$ is greater in the presence of NSI if $K_{-}>0$ and less if $K_{-}<0$. When $K_{-}<0$, the dependence on $K_{+}$is very weak, it is completely negligible when $K_{-}=0$ and becomes somewhat stronger when $K_{-}>0$. For convenience, in Fig. 9 we use the common value $\Delta m_{31}^{2}=(2.32+0.023=2.343) \times 10^{-3} \mathrm{eV}^{2}$, the value at the minimum when NSI are zero, for all the points. In fact the minimum value of $\Delta \chi^{2}$ shows some dependence on $K_{+}$and $K_{-}$, though the qualitative features of the landscape shown in the figure do not change.

One can make a correspondence between the $\Delta \chi^{2}$ values at fixed $a=0.03$ and $b=0$ energy uncertainties while NSI parameters $K_{+}$and $K_{-}$vary, and the values that $\Delta \chi^{2}$ takes on in the $K_{+}=K_{-}=0$ SMM case while $a$ and $b$ vary. For example, with $K_{+}$in the range from $0.0-0.02, K_{-}=0.04$ and $a=0.03$ and $b=0$, the $\Delta \chi^{2} \simeq 6.2$ is about the same as the IH $\Delta \chi_{\min }^{2}$ value for $a=0.02, b=0.01$ at $50 \mathrm{~km}$ in Fig. 7 of [22], compared to the $\Delta \chi^{2} \simeq 3.5$ shown in Fig. 6 of 22] for $a=0.03$ and $b=0$. This improved sensitivity is driven by the positive value of $K_{-}$, as indicated in our Fig. 9. Conversely, as $K_{-}$takes on negative values, the sensitivity is degraded. Combined with hints of NSI in fits to the event spectrum or hints from other, independent experiments, an anomalously low or high sensitivity to the MH may indicate that NSI are at work.

We do not pursue this in detail here, since our limited goal is to identify points where planned medium baseline reactor neutrino precision experiments can be sensitive to NSI and points where NSI mimic the effects of uncertainties in the value of input parameters. The latter degeneracies make the precision determination of the MH and of SMM parameters model dependent. 


\section{SUMMARY AND CONCLUSIONS}

Current limits on NSI parameters are tight enough and the recently measured value of reactor $\overline{\nu_{e}}$ disappearance probability is large enough that NSI effects can be analyzed transparently at leading order in the parameters. In this framework, we identified two effective NSI parameters that play a role in short and medium baseline neutrino disappearance analysis, $K_{+}$and $K_{-}$, which are combinations of the complex flavor violating NSI coefficients and, in the case of $K_{+}$, the standard mixing matrix $\mathrm{CP}$-violating phase $\delta$. The short baseline experiments [1 $\left[\begin{array}{l}3 \\ ]\end{array}\right.$, constrain a combination of $\sin ^{2}\left(2 \theta_{13}\right)$ and $K_{+}$, and we used this constraint as an essential input to our analysis of consequences for medium baseline $\overline{\nu_{e}}$ disappearance experiments that are in the planning stage, aimed at determining the neutrino $\mathrm{MH}$ and the precision measurement of mixing angles and magnitudes of mass-squared differences.

In Sec.III.A we outlined the constraint linking $K_{+}$and $\sin ^{2}\left(2 \theta_{13}\right)$, essentially reducing the analysis of the medium baseline experiments to a two parameter problem, either $K_{+}$and $K_{-}$or $\sin ^{2}\left(2 \theta_{13}\right)$ and $K_{-}$. The constraint is shown in Fig. 1. In Sec.III.B, we showed that the event rate spectra at fixed baseline, $50 \mathrm{~km}$ being the default value throughout our analysis, are sensitive to the NSI parameters for given, fixed values of $\sin ^{2}\left(2 \theta_{12}\right)$ and $\sin ^{2}\left(2 \theta_{13}\right)$, even with generous energy uncertainties allowed. When $\sin ^{2}\left(2 \theta_{12}\right)$ was varied within its $1 \sigma$ range with no NSI present, we found the effects were mimicked by NSI parameters varied in the same range while $\sin ^{2}\left(2 \theta_{12}\right)$ was held fixed at its central value, Figs. 2 and 3, Outside this range, the variation in NSI starts to show up, as we show in Fig. 4 , This degeneracy was then explored further in Sec.IV. In Sec.III.B.2, the long oscillation length term, sensitive to the probability factor $P_{21}$, was determined to depend only on $\sin ^{2}\left(2 \theta_{12}\right)$ and $K_{-}$, the dependence on $K_{+}$being entirely negligible, simplifying the statistical analysis in Sec.IV. The short oscillation length, MH sensitive term is controlled by the probability factor $P_{32}$, which we showed depends linearly on $K_{-}$, with a coefficient that depends only weakly on $K_{+}$through its constraint with $\sin ^{2}\left(2 \theta_{13}\right)$. The impact of NSI is not as dramatic on the MH sensitive terms as on the scale of the rate spectrum, as indicated by comparing Figs. 3. left panel, and 5, right panel. Nonetheless, with sufficient statistics and reduction of systematic uncertainties, the distinction can be made [22]. However, the effect of the NSI is degenerate with variation in $\sin ^{2}\left(2 \theta_{13}\right)_{e f f}$, emphasizing the need for precision determination of $\sin ^{2}\left(2 \theta_{13}\right)$ independent of reactor neutrino experiments [29].

Following up on these considerations in Sec.IV.A, we varied the value of $\sin ^{2}\left(2 \theta_{12}\right)$ within its $1 \sigma$ range in the NSI fit function, while adopting as "data" the SMM with central values of input parameters. Modulo the "pull" term, which penalizes the fit by one unit of $\chi^{2}$ at $1 \sigma, 4$ units at $2 \sigma$ and so forth, we found a continuum of very nearly degenerate $\chi^{2}$ minima with values typically of the order $10^{-4}$ to $10^{-5}$. The spread in $K_{-}$values at the minima is roughly from -0.025 to +0.025 as $\sin ^{2}\left(2 \theta_{12}\right)$ runs over its $1 \sigma$ uncertainty, $0.857 \pm 0.024$. The effects of varying other parameters were negligible. The bounds on the $K_{-}$parameter follow the bounds on the input uncertainties in $\sin ^{2}\left(2 \theta_{12}\right)$ quite closely. This observation has important consequences: the tighter the input uncertainties, the higher the sensitivity to the NSI parameter $K_{-}$, so doubling the precision doubles the sensitivity to NSI parameters!

Finally, in Sec.V we investigated the influence of NSI on the statistical discrimination between NH and IH in medium baseline experiments. Generalizing the pattern of analysis of Ref. [22], we showed that, for a given statistical and systematic energy determination uncertainties, the allowed NSI parameter ranges can either enhance or suppress the sensitivity expectations based on the SMM analysis. Improved, independent determination of $\sin ^{2}\left(2 \theta_{13}\right)$ can improve the sensitivity to NSI parameter $K_{-}$in the $\mathrm{MH}$ determination in medium baseline experiments, as remarked in Sec.III.B.1. Moreover, it can provide sensitivity to the parameter $K_{+}$in the short baseline experiments.

We conclude that the planned medium baseline, reactor neutrino experiments to determine the $\mathrm{MH}$ and to make precision measurements of neutrino parameters are also good probes of NSI as the measurements of $\sin ^{2}\left(2 \theta_{12}\right)$ and $\sin ^{2}\left(2 \theta_{13}\right)$ in independent experiments such as SNO+ [51], Hyper-Kamiokande [52] and T2K [6, 53] become more precise. This will enable one to restrict the degeneracies pointed out in Secs. III and IV and increase sensitivity to NSI [29]. In particular, the overall spectrum statistics provide a method for detecting the presence of NSI at levels below those available in the literature. This diagnostic is not sensitive to the sign of the relevant parameter. If there is evidence from the gross spectral features that NSI are present, the short oscillation length pattern that reveals the $\mathrm{MH}$ also provides additional information on its sign, which determines whether the MH signal is suppressed or enhanced.

\section{Acknowledgments}

D. W. M would like to thank the Physics Department of CIIT Islamabad for hosting his visit while this work was begun. The visit was supported by the Higher Education Commission (HEC) of Pakistan. A. N. K. would like to thank The University of Kansas (KU) Particle Theory Group for kind hospitality during his visit to KU, the HEC for support for his graduate studies and for his visit to KU under the Indigenous Ph.D. Fellowship Program (5000 Fellowships) Batch-IV and International Research Support Initiative Program. The authors benefitted from detailed 
communications with Dr. Shao-Feng Ge and Dr. Yoshitaro Takaesu. D.W. M. and A. N. K received support from DOE Grant. No. De-FG02-04ER41308.

[1] Y. Abe et al. [Double Chooz Collaboration], Phys. Rev. Lett. 108, 131801 (2012); Y. Abe et al. [Double Chooz Collaboration], Phys. Rev. D 86, 052008 (2012).

[2] F. An et al. [Daya Bay Collaboration], Phys. Rev. Lett. 108, 171803 (2012).

[3] J. Ahn et al. [RENO Collaboration], Phys. Rev. Lett. 108, 191802 (2012).

[4] F. An et al. [Daya Bay Collaboration], Chin. Phys. C37, 011001 (2013).

[5] P. Adamson et al. [MINOS Collaboration], Phys. Rev. Lett. 107, 181802 (2011).

[6] K. Abe et al. [T2K Collaboration], Phys. Rev. Lett. 107, 041801 (2011).

[7] J. Kopp, M. Lindner, T. Ota and J. Sato, Phys. Rev. D 77, 013007 (2008).

[8] T. Ohlsson and H. Zhang, Phys. Lett. B 671, 99 (2009).

[9] R. Leitner, M. Malinsky, B. Roskovec and H. Zhang, J. High Energy Phys. 12 (2011).

[10] C. Biggio, M. Blennow and E. Fernández-Martínez, JHEP 08, 090 (2009); arXiv:0907.0097v3 [hep-ph].

[11] S. Petcov and M. Piati, Phys. Lett. 533, 94 (2002).

[12] S. Petcov and M. Piati, Phys. Rev. D 68, 113006 (2003).

[13] J. Learned, S. Dye, S. Pakvasa, and R. Svoboda, Phys. Rev. D 78, 071302 (2008).

[14] H. Minakata, H. Nunokawa, S. Parke and R. Funchal, Phys. Rev. D 76, 053004 (2007).

[15] L. Zhan, Y. Wang, J. Cao and L. Wen, Phys. Rev. D 79, 073007 (2009).

[16] X. Qian, D. Dwyer, R. McKeown, P. Vogel, W. Wang and C. Zhang, Phys. Rev. D 87, 033005 (2013); arXiv:1208.1551v2 [physics.ins-det] (2012).

[17] E. Ciuffoli, J. Evslin and X. Zhang, JHEP 1303, 016 (3013); arXiv:1208.1991, [hep-ph] (2012).

[18] P. Ghoshal and S. Petcov, JHEP 1209, 115; arXiv:1208.6473 [hep-ph] (2012).

[19] E. Ciuffoli, J. Evslin and X. Zhang, JHEP 1212, 004; $\operatorname{arXiv:1209.2227}$, [hep-ph] (2012).

[20] E. Ciuffoli, J. Evslin and X. Zhang, arXiv:1302.0624v1 [hep-ph] (2013).

[21] X. Qian, A. Tan, W. Wang, J. Ling, R. McKeown and C. Zhang, Phys. Rev. D 86, 113011 (2012); arXiv:1210.3651 [physics.ins-det] (2012).

[22] S-F. Ge. K. Hagiwara, N. Okamura and Y. Takaesu, JHEP 1305, 131 (2013), arXiv:1210.8141v2 [hep-ph] (2012). This reference provides an efficient method for estimating the statistical uncertainty of the $\chi^{2}$ sensitivity analysis.

[23] J. Cao, talk at VCI, Feb. 2013.

[24] Y-F. Li, J Cao, Y. Wang and L. Zhan, arXiv:1303.6733v1 [hep-ex] (2013).

[25] Y. Wang, talk at NeuTel, July 2013

[26] RENO-50 Collaboration in International Workshop on RENO-50: toward Neutrino Mass Hierarchy, 2013

[27] A. Gando et al. Phys. Rev. D 83, 052002 (2011).

[28] T. Ohlsson, Rep. Prog. Phys. 76, 044201 (2013).

[29] Analysis of solar experiments including the NSI at the source $\left({ }^{8} \mathrm{~B}\right.$ decay for instance) but different NSI at the detector, or no NSI at the detector, combined with the current analysis would be necessary. The neutrino detection in the purely leptonic or neutral current modes would satisfy this requirement. To the best of our knowledge, only NSI in propagation and/or detection have been done for the solar neutrino case. See, for example [28] and references therein. This is a topic for further research and goes beyond the scope of this paper.

[30] Y. Grossman, Phys. Lett. B 359, 141 (1995).

[31] L. Johnson and D. McKay, Phys. Lett. B 433, 335 (1998).

[32] S. Bergmann and Y. Grossman, Phys. Rev. D 59, 093005 (1999).

[33] L. Johnson and D. McKay, Phys. Rev. D 61, 113007 (2000).

[34] M. Gonzalez-Garcia, Y. Grossman, A. Gusso and Y. Nir, Phys. Rev. D 64, 096006 (2001).

[35] "Probing Lepton Flavor Violation", D. McKay, and L. Johnson, in Proceedings of PASCOS99, Lake Tahoe, K. Cheung, J. Gunion and S. Mrenna, Eds. World Scientific, Singapore, 2000. pp 204-207.

[36] A more general form with flavor dependent right-handed coefficients is given in [10]. We simplify to left-handed helicity in the work here, so our $K_{i j}$ are equivalent to $\epsilon_{i j}^{u d L}$ in [10].

[37] In any case, the normalization is absorbed into a redefinition of the effective Fermi constant, whose experimental constraints via universality show up in the model independent bounds on the NSI parameters [10] we use here.

[38] Note that the $P_{i j}$ notation used in [15] absorbs the factors $\sin ^{2} x_{i j}$ as well.

[39] The sign and value of $x_{21}$ have been determined by solar neutrino experiments.

[40] J. Beringer et al. [Particle Data Group], Phys. Rev. D 86, 010001 (2012).

[41] As remarked earlier, we assume that phase "accidents" $\cos \left(\delta+\phi_{e \mu}\right)=\cos \left(\delta+\phi_{e \tau}\right)=0$ or $\cos \phi_{e \mu}=\cos \phi_{e \tau}=0$ don't happen.

[42] This result is essentially the same as that of Eq. (28) in 7] for the case of same physics at source and detector, and as a linear approximation to Eqs.(17) and (18) of [8] or Eqs. (3.1) and (3.2) of [9]. A comparison between our Fig. 11 and the right-hand-side of Fig. 1 of [8] can be made roughly by fixing $\tilde{\theta}_{13}$ at 8.5 degrees, which corresponds to $\sin ^{2}\left(\theta_{13}\right)_{e f f}=0.089$, and comparing the range of $\theta_{13}$ values for $|\epsilon|=0.01$ to that corresponding to difference between its value at $K_{+}=-0.01$ 
and +0.01 in our Fig. 1 which is between about 8 degrees and 9 degrees. There is agreement between the two figures, as far as we can tell from Fig. 1 in [8].

[43] Unless we specify otherwise, we will assume maximal 2-3 mixing.

[44] "NOvA Proposal to Build a 30 Kiloton Off-Axis Detector to Study Neutrino Oscillations in the Fermilab NuMi Beamline", D. Ayers et al. [The NOvA Collaboration], arXiv hep-ex/0503053v1; "The NOvA Experiment: Status and Outlook", R. Patterson for the NOvA Collaboration, to appear in the proceedings of Neutrino 2012, arXiv:1209.0706v1 [hep-ex].

[45] "Interim Design Report", R.J. Abrams et al., arXiv:1112.2853 v1 [hep-ex]; "Enabling Intensity and Energy Frontier Science with a Muon Accelerator Facility in the U.S.:, A White Paper Submitted to the 2013 U.S. Community summer Study of the Division of Particles and Fields of the American Physical Society", Contributed by the U.S. MAP and Associated Collaborators, J-P Delahaye et al. Eds., arXiv:1308.0494 [physics.acc-phy]

[46] P. Vogel and J. Engel, Phys. Rev. D 39, 3378, (1989).

[47] P. Vogel and J. Beacom, Phys. Rev. D 60, 053003 (1999).

[48] Varying the value of $\sin ^{2}\left(2 \theta_{13}\right)_{\text {eff }}$ within its $1 \sigma$ range has barely noticeable effect on the spectral curves - of the order of the widths of the lines in the figure.

[49] B. Aharmin et al. [The SNO Collaboration], Phys. Rev. C 81, 055504 (2010); Phys. Rev. C 88, 025501 (2013).

[50] In the extreme case of very large NSI parameter values, the overall sign of $P_{32}$ can change and reverse the expected pattern of $\mathrm{NH}$ vs. IH oscillations. Within the constrained range of parameters, roughly where $\left|K_{ \pm}\right| \leq 0.05$, there is no point where it becomes zero or negative, so the NSI cannot reverse the expected signs of NH and IH. At the point $(-0.1,-0.2)$, more than 4 standard deviations outside the constrained range for the NSI parameters and outside of the linear region, $P_{32}=0$, and it becomes negative for values of $K_{-}<-0.2$ with $K_{+} \leq-0.1$.

[51] I. Coulter [SNO+ Collaboration], "Event reconstruction and background rejection in the SNO+ experiment", IOP talk, Liverpool, April 2013.

[52] K. Abe et al. [The Hyper-Kamiokande Collaboration], "Letter of Intent: The Hyper-Kamiokande Experiment - Detector Design and Physics Potential", arXiv:1109.3262v1 [hep-ex] (2011).

[53] M. Wilking [T2K Collaboration], "New Results from the T2K Experiment: Observation of $\nu_{e}$ Appearance from a $\nu_{\mu}$ Beam", EPS Conference talk, July 19, 2013. 